\title{
Evaluation of Antidiabetic Activities of Scorzonera Species on Alloxan-Induced Diabetic Mice
}

\author{
Ayse Arzu Sakul ${ }^{1}$ (D) Ekin Kurtul ${ }^{2}\left(\mathbb{D}\right.$, Hanefi Ozbek $^{3}$ (D), Neriman Ipek Kirmizi ${ }^{1}$ (D), Bade Cevriye Bahtiyar ${ }^{4}(\mathbb{D}$, \\ Gulcin Saltan Iscan ${ }^{2}$ (D), Ozlem Bahadir Acikara ${ }^{2}$ (D) \\ ${ }^{1}$ Istanbul Medipol University, Faculty of Medicine, Department of Pharmacology, Istanbul, Turkey. \\ ${ }^{2}$ Ankara University, Faculty of Pharmacy, Department of Pharmacognosy, Ankara, Turkey. \\ ${ }^{3}$ Izmir Bakircay University, Faculty of Medicine, Department of Pharmacology, Izmir, Turkey \\ ${ }^{4}$ Istanbul Medipol University, Vocational School of Health Services, Istanbul, Turkey. \\ Correspondence Author: Neriman Ipek Kirmizi \\ E-mail: nikirmizi@medipol.edu.tr \\ Received: 21.09.2020 Accepted: 04.02.2021
}

\begin{abstract}
Objective: In Turkish folk medicine, different species of Scorzonera (Asteraceae) have been used in the treatment of various disorders. The study aimed to investigate antidiabetic activity of Scorzonera species and if rutin is the primary component responsible of this activity.

Methods: The extracts of aerial parts of Scorzonera tomentosa, S. mollis ssp. szowitsii, S. suberosa ssp. suberosa, S. eriophora, S. acuminata, S. sublanata and S. cana var. jacquiniana were used in the experiment. Additionally, rutin, which has been analysed and detected by using HPLC qualitatively and quantitatively in some Scorzonera species, was also tested for its antidiabetic activity in the same conditions. An alloxaninduced diabetic mice test model was used in order to verify antidiabetic activity. Antidiabetic activities of the 9 groups ( $\mathrm{n}=5$; each) were measured at four different times: before Scorzonera extracts and rutin treatment and after 1, 2 and 4 hours of treatments.

Results: S. sublanata extract exhibited the highest antidiabetic activity and at $100 \mathrm{mg} / \mathrm{kg}$ dose, it significantly reduced blood glucose levels measured after 1, 2 and 4 hours of treatments when compared to isotonic saline solution group (diabetic control group). S. cana var. jacquiniana extract also displayed notable decrease after 4 hours of treatment. Significant lowering effect on blood glucose level was also observed by treatment with rutin in all tested times at $100 \mathrm{mg} / \mathrm{kg}$ i.p. injection. According to the HPLC analyses the highest rutin content was determined in the S. acuminata aerial parts.

Conclusion: Rutin content and the antidiabetic activity of the plant extracts were not correlated as displayed in this present study. Further studies should be performed to reveal responsible compunds for antidiabetic activity.
\end{abstract}

Keywords: Alloxan, antidiabetic activity, diabetes, rutin, Scorzonera

\section{INTRODUCTION}

Diabetes is a chronic metabolic disorder characterized by stable high levels of blood glucose, which is known as the seventh leading cause of death in the world and affects 100 million people annually. Factors such as aging, obesity, physical inactivity, population growth and urbanization gradually lead to a steady increase in the number of patients with diabetes. In the year 2000 , the prevalence of diabetes worldwide among adults was estimated as approximately 171 million. This number increased to 422 million in 2014, and it is expected that in year 2030, approximately 366 million people will suffer from diabetes worldwide $(1,2)$.

Diabetes is closely associated with other diseases such as hypertension, cardiovascular diseases, atherosclerosis, peripheral vascular diseases, and insufficient control generally results in many complications in the vascular system, kidneys, retinas, lenses, peripheral nerves and skin as well as overall quality of life $(3,4)$. Therefore, still there has been a growing interest in drug development for diabetes, especially phytochemicals derived from plants (5).

Scorzonera L. genus belonging to the Asteraceae family is widespread in more arid regions of Eurasia and northern Africa with about 160 species. Scorzonera species grow naturally by 52 species in Turkey and 31 of which are endemic (6). Scorzonera hispanica, the most common species of Scorzonera in Europe, which was consumed by the ancient Romans and Greeks has been cultured since the sixteenth century for medicinal purposes and as a vegetable. In the middle age, this plant was used as a tonic as well as for treatment of snakebites. Nowadays, especially in Belgium, France and Holland, it is cultured for consumption as a vegetable. Scorzonera species are used in the treatment of various diseases, including the common cold, appetite and pectoral problems, as a mucolytic, diuretic, antipyretic and diaphoretic (7-10). There is also information about its use for digestive problems as well as in the treatment and 
prevention of diabetes $(11,12)$. In Turkey, the young shoots and the leaves of some Scorzonera species are consumed either raw or cooked. In addition, they are recorded in Turkish folk medicine as treatments for atherosclerosis, high blood pressure, rheumatoid arthritis, kidney diseases and diabetes (7). Some of the species belonging to this genus are also used for their antidiabetic activities in Turkish folk medicine. These include Scorzonera mollis M. Bieb. subsp. szowitzii (DC.), S. semicana DC., S. cinerea Boiss., Scorzonera latifolia (Fisch. \& C.A.Mey.) DC. var. latifolia (13-15). Although, there are various studies revealing the antidiabetic effect of rutin (16, 17), none of these studies has been found to be related to the antidiabetic activities of Scorzonera species selected for this present study.

As diabetes is a progressive disease, besides insulin, there is still a need to find effective compounds for the treatment. Medicinal plants are good sources of new drugs and many of the currently available drugs have been derived directly or indirectly from them $(1,2,5)$. In light of the traditional usage of Scorzonera species for the treatment of diabetes, this study investigated aerial parts of some Scorzonera species including $S$. acuminata, S. cana var. jacquiniana, $S$. eriophora, S. mollis ssp. szowitsii, S. sublanata, S. suberosa ssp. suberosa, S. tomentosa for their potential antidiabetic activity. Moreover, this study tried to establish rutin as the responsible component for antidiabetic activity.

\section{METHODS}

\subsection{Plant Material}

Scorzonera species were collected from different parts of Anatolia, Turkey in their flowering time. Prof. Hayri Duman, a plant taxonomist from the Gazi University, confirmed the taxonomic identification of the plants. Voucher specimens are kept in the herbarium of Ankara University, Faculty of Pharmacy (Table 1).

Table 1. List of Scorzonera species collected for the study

\begin{tabular}{lll}
\hline Species & Collection Place & $\begin{array}{l}\text { Herbarium } \\
\text { number }\end{array}$ \\
\hline S. acuminata Boiss. & Yumakli village, Cankiri, 2010 & AEF 25938 \\
\hline $\begin{array}{l}\text { S. cana (C.A. Meyer) } \\
\text { Hoffm. var. jacquiniana } \\
\text { (W. Koch) Chamberlain }\end{array}$ & Camlidere town, Ankara, 2008 & AEF 23834 \\
\hline S. eriophora DC. & Cubuk town, Ankara, 2007 & AEF 23832 \\
\hline $\begin{array}{l}\text { S. } \text { mollis Bieb. subsp. } \\
\text { szowitsii (DC.) }\end{array}$ & Kizilcahamam town, Ankara, 2006 & AEF 23844 \\
Chamberlain & & \\
\hline $\begin{array}{l}\text { S. suberosa C. Koch } \\
\text { subsp. suberosa }\end{array}$ & Pinarbasi town, Kayseri, 2006 & AEF 23843 \\
\hline S. sublanata Lipschitz & Kizilcahamam town, Ankara, 2010 & AEF 25937 \\
\hline S. tomentosa L. & Akdagmadeni town, Yozgat, 2005 & AEF 23841 \\
\hline
\end{tabular}

\subsection{Preparation of the Extract}

Dried and powdered aerial parts of the plant material were extracted with methanol:water $(80: 20 \mathrm{v} / \mathrm{v})$ mixture at room temperature through 8 hours by continuous stirring which was followed by maceration during 16 hours for 3 days. Each extract was filtered from filter paper and concentrated to dryness under reduced pressure and low temperature (40$50^{\circ} \mathrm{C}$ ) on a rotary evaporator to yield crude extracts.

\subsection{Isolation of Rutin}

Rutin was isolated from S. acuminata aerial parts. S. acuminata aerial parts (475.79 g) were extracted with methylalcohol at room temperature for 24 hours followed by extraction in ultrasonic bath for 1 hour. After 5 times this extraction techniques were applied, all extracts were filtered and evaporated under vacuum $\left(40-50^{\circ} \mathrm{C}\right)$ on a rotary evaporator. Obtained crude extract $(72.74 \mathrm{~g})$ suspended in water and extracted with petroleum ether and ethylacetate respectively by using liquid-liquid extraction techniques. The ethylacetate part (10.56 g) was subjected to column chromatography on silica gel and eluted with ethylacetate: methanol:water (100:13.5:10) mixture to obtain 52 fractions. Fraction 35 gave a yellow amorphous precipitate. After filtration, compound purity was checked with HPLC and $\mathrm{MS}$, as well as with NMR $\left({ }^{1} \mathrm{H}-\right.$ and $\left.{ }^{13} \mathrm{C}-\mathrm{NMR}\right)$, analyses were conducted for structure elucidation (Table S1). For HPLC analysis as well as antidiabetic activity assays, isolated compound which was detected in enough purity was used.

\subsection{HPLC Analysis}

HPLC analyses were carried out using Agilent LC 1100 model chromatograph (Agilent Technologies, California, USA). The diode array detector (DAD) was set at a wavelength of $254 \mathrm{~nm}$ and peak areas were integrated automatically by computer using Agilent Software. The chromatograms were plotted and processed using Agilent software. Separation was carried out using a Supelcosil $(250 \mathrm{~mm} \times 4.6 \mathrm{~mm} ; 5 \mu \mathrm{m})$ column. The mobile phase was made up of acetonitrile $(A)$ and water $(B)$ in gradient elution: initial 0 min, $A-B$ (8:92, $\mathrm{v} / \mathrm{v})$, then $0-10 \mathrm{~min}$, linear change from $A-B(8: 92, \mathrm{v} / \mathrm{v})$ to $A-B$ (18:82), $10-20 \mathrm{~min}$, there is isocratic flow $A-B(18: 82)$ and the linear gradient elution is from $A-B(20: 80)$ to $A-B$ (22:78) with the range of $20-45 \mathrm{~min}$. This was followed changing $A-B(22: 78)$ from $45 \mathrm{~min}$ to $55 \mathrm{~min}$. The flow rate was $0.7 \mathrm{~mL} / \mathrm{min}$ and column temperature were maintained at $40^{\circ} \mathrm{C}$. The sample injection volume was $10 \mu \mathrm{L}$.

\subsection{Preparation of Standard Solutions and Calibration}

Standard stock solution for rutin was prepared as $1 \mathrm{mg} /$ $\mathrm{mL}$. Rutin was weighed in $10 \mathrm{~mL}$ volumetric flask, dissolved in methanol:water (80:20) mixture and adjusted to the final volume separately. Six different concentration levels ( $0.01 \mathrm{mg} / \mathrm{mL}, 0.02 \mathrm{mg} / \mathrm{mL}, 0.05 \mathrm{mg} / \mathrm{mL}, 0.1 \mathrm{mg} / \mathrm{mL}, 0.2 \mathrm{mg} /$ $\mathrm{mL}$, and $0.5 \mathrm{mg} / \mathrm{mL}$ ) were prepared by diluting the stock solution. Triplicate $10 \mu \mathrm{L}$ injections were performed for each 
standard solution. Peak area of each solution was plotted against the concentration to obtain the calibration curves.

\subsection{Validation Procedure}

\subsubsection{Limit of detection and quantification}

Limit of detection (LOD) and limit of quantification (LOQ) were established at a signal to noise ratio $(\mathrm{S} / \mathrm{N})$ of 3 and 9 , respectively. LOD and LOQ concentrations were experimentally verified by 6 injections of rutin. LOD and LOQ levels were also determined as 0.13 and $0.45 \mu \mathrm{g} / \mathrm{mL}$ respectively.

\subsubsection{Precision}

Precision tests were performed by the evaluation of intra-day variations of the same standard solution of rutin the LOQ level. The intra-day precision was determined by analyzing the same samples six times in a single day. The inter-day precision was determined three different days in triplicates of three different concentration injections. The results of precision tests were expressed as the relative standard deviations (RSDs) of the retention time $\left(R_{t}\right)$ and peak area $\left(P_{a}\right)$ for rutin.

\subsection{Animals}

Adult female Balb/C strain mice (22-30 g) obtained from Istanbul Medipol University Regenerative and Restorative Medical Center (REMER), were used for this experiment. The animals were housed in standard cages $(48 \mathrm{~cm} \times 35 \mathrm{~cm}$ $\times 22 \mathrm{~cm})$ at room temperature $\left(22 \pm 2{ }^{\circ} \mathrm{C}\right)$, with artificial light from 7.00 am to $7.00 \mathrm{pm}$, and provided with pelleted food and water ad libitum. Ethics Committee of Istanbul Medipol University approved the study protocol (01/02/2017-03).

\subsection{Antidiabetic Activity Assay}

An alloxan-induced test model was used to evaluate antidiabetic activity $(3,18,19)$. Mice were kept without food for 18 hours before the alloxan treatment. Alloxan was applied in an isotonic saline solution by i.p. (intraperitoneal) administration ( $150 \mathrm{mg} / \mathrm{kg}$ of body weight) three times over a 48-hour period. Mice were kept without food following the last alloxan treatment, and blood glucose levels were measured. Previous studies have shown that blood glucose of healthy mice should be below $125 \mathrm{mg} / \mathrm{dL}(20,21)$. In this study mice with blood glucose levels of $200 \mathrm{mg} / \mathrm{dL}$ and higher were subjected to further studies as diabetic animals. Diabetic mice were divided into nine groups in order to determine the antidiabetic activities of Scorzonera extracts (all extracts were dissolved in an isotonic saline solution). Animals were assigned to the following groups ( $n=5$, for each): Group 1, the control group, received isotonic saline solution (ISS) $0.1 \mathrm{~mL}$; other groups received $S$. tomentosa, $S$. mollis ssp. szowitsii, S. suberosa ssp. suberosa, S. eriophora, S. acuminata, S. sublanata, S. cana var. jacquiniana extracts $100 \mathrm{mg} / \mathrm{kg}$ of body weight i.p., respectively. This procedure was followed by rutin investigation (in total 9 animal groups $[n=5$, for each] was evaluated). Rutin was dissolved in an isotonic saline solution and administered $100 \mathrm{mg} / \mathrm{kg}$ of body weight i.p. The dosage of the plant extracts $(22,23)$ and rutin (17) were determined according to the results of previous studies. Animals were sacrificed in the end of the study.

Following the administration of the tested extracts and rutin, blood samples collected from the tail at the $1^{\text {st }}, 2^{\text {nd }}$ and $4^{\text {th }}$ hours were measured for their glucose concentrations, using an Accu-Check sugar strip, employing the glucose-oxidaseperoxidase method.

\subsection{Statistical Analyses}

Statistical analysis was carried out using SPSS 22.0 software. The results were reported as mean \pm standard error of mean (SEM). A one-way analysis of variance (post-hoc Dunnett-t test) was used for statistical analyses. Probability levels of less than 0.05 were considered as significant.

\section{RESULTS}

The aerial parts of Scorzonera tomentosa, S. mollis ssp. szowitsii, S. suberosa ssp. suberosa, S. eriophora, S. acuminata, S. sublanata and S. cana var. jacquiniana were tested for their antidiabetic activities for the first time in current study. Scorzonera extracts were tested for their potential antidiabetic activities using alloxan-induced diabetic animals. Blood glucose levels were measured at four different times: before Scorzonera extracts treatment and after 1, 2 and 4 hours of treatment. All of the measured levels of blood glucose are presented in Table 2. According to the results, $S$. sublanata was associated with significantly decreased blood glucose levels after 1, 2 and 4 hours of treatment; these were determined as follows: $207.20 \pm 28.45 ; 200.80 \pm 43.88$ and $174.00 \pm 38.77 \mathrm{mg} / \mathrm{dL}$, when compared to the isotonic saline group (diabetic control group) $(p<0.05)$. Additionally, S. cana var. jacquiniana caused notable decreasing in blood glucose levels after 4 hours of treatment as shown in Table 2, $232.80 \pm 49.80 \mathrm{mg} / \mathrm{dL}$ respectively when compared to the diabetic control group $(p<0.05)$.

A similar research procedure was applied to diabetic mice, which received rutin compound as one of the constituents of some Scorzonera species. Rutin was found to have significant antidiabetic activity, and blood glucose levels decreased significantly to $307.20 \pm 40.73$ after 1 hour, $236.40 \pm 50.48$ after 2 hours and $190.60 \pm 52.69 \mathrm{mg} / \mathrm{dL}$ after 4 hours of treatment compared with diabetic control group.

Phytochemical structure of the tested extracts was also investigated by HPLC analysis with this research. Mainly flavonoids as well as chlorogenic acid derivatives have been detected (Figure S1-S7). Furthermore, content of the rutin (Figure 1) which was isolated from the S. acuminata aerial parts, of the tested extracts were determined qualitatively and quantitatively (Table 3 ) to reveal whether there is a relationship between rutin content and antidiabetic activity. Calibration curve of the rutin was exhibited as Figure 2. According to the HPLC results, S. acuminata aerial parts contain higher amount of rutin than the other Scorzonera 
species which was determined as $442.69 \pm 24.33 \mu \mathrm{g} / \mathrm{mL}$. Rutin was also detected as $23.30 \pm 0.067$ and $53.46 \pm 1.59 \mu \mathrm{g} /$ $\mathrm{mL}$ for S. eriophora and S. mollis ssp. szowitzii aerial parts, respectively. S. sublanata contains rutin in trace amount while the remaining extracts were found to lack rutin (Table 3).

Table 2. Blood glucose levels of Scorzonera treated alloxan-induced diabetic mice

\begin{tabular}{|c|c|c|c|c|}
\hline \multirow[t]{3}{*}{ Groups } & \multicolumn{4}{|c|}{$\begin{array}{l}\text { Blood sugar levels (mg/dL) } \\
{[\text { Mean } \pm \text { Standart Error of Mean] }}\end{array}$} \\
\hline & \multirow{2}{*}{$\begin{array}{l}\text { Before Scorzorena } \\
\text { treatment }\end{array}$} & \multicolumn{3}{|c|}{ After Scorzorena treatment } \\
\hline & & 1. hour & 2. hour & 4. hour \\
\hline Control (ISS) & $470.60 \pm 23.61$ & $472.20 \pm 13.94$ & $493.80 \pm 15.55$ & $494.20 \pm 27.32$ \\
\hline S. acuminata & $479.20 \pm 28.16$ & $466.80 \pm 29.13$ & $429.20 \pm 42.48$ & $415.60 \pm 71.70$ \\
\hline S. cana var. jacquiniana & $361.60 \pm 31.80$ & $407.60 \pm 30.77$ & $376.40 \pm 42.36$ & $232.80 \pm 49.80^{*}$ \\
\hline S. eriophora & $331.00 \pm 45.93$ & $372.20 \pm 49.38$ & $340.60 \pm 54.19$ & $300.20 \pm 61.05$ \\
\hline S. mollis ssp. szowitsii & $412.00 \pm 27.60$ & $413.00 \pm 33.02$ & $430.40 \pm 38.63$ & $342.20 \pm 55.24$ \\
\hline S. suberosa ssp. suberosa & $418.20 \pm 27.06$ & $371.80 \pm 70.06$ & $297.00 \pm 70.56$ & $308.60 \pm 65.19$ \\
\hline S. sublanata & $312.20 \pm 31.27$ & $207.20 \pm 28.45^{*}$ & $200.80 \pm 43.88^{*}$ & $174.00 \pm 38.77^{*}$ \\
\hline S. tomentosa & $405.00 \pm 37.56$ & $361.60 \pm 50.73$ & $362.80 \pm 59.87$ & $277.00 \pm 58.56$ \\
\hline Rutin & $368.80 \pm 42.19$ & $307.20 \pm 40.73$ & $236.40 \pm 50.48^{*}$ & $190.60 \pm 52.69^{*}$ \\
\hline
\end{tabular}

Post-hoc Dunnett-t test; * comparision with saline group ( $p<0.05)$

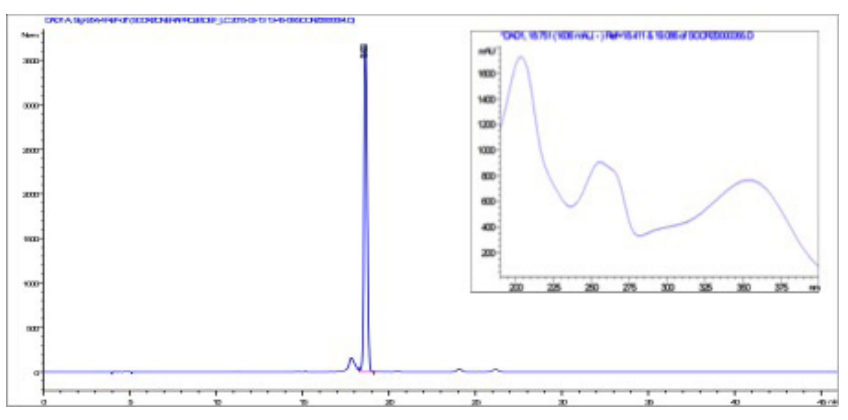

Figure 1. HPLC chromatogram and UV spectrum of isolated rutin

Table 3. Rutin content of the tested Scorzonera species

\begin{tabular}{l|l}
\hline Species & $\begin{array}{l}\text { Rutin ( } \mu \mathrm{g} / 100 \text { mg plant material) } \\
{[\text { Mean } \pm \text { Standart Deviation] }}\end{array}$ \\
\hline S. acuminata & $442.69 \pm 24.33$ \\
\hline S. cana var. jacquiana & - \\
\hline S. eriophora & $23.30 \pm 0.067$ \\
\hline S. mollis ssp. szowitzii & $53.46 \pm 1.59$ \\
\hline S. suberosa ssp. suberosa & - \\
\hline S. sublanata & tr. \\
\hline S. tomentosa & - \\
\hline
\end{tabular}

tr: trace (under limit of quantification)

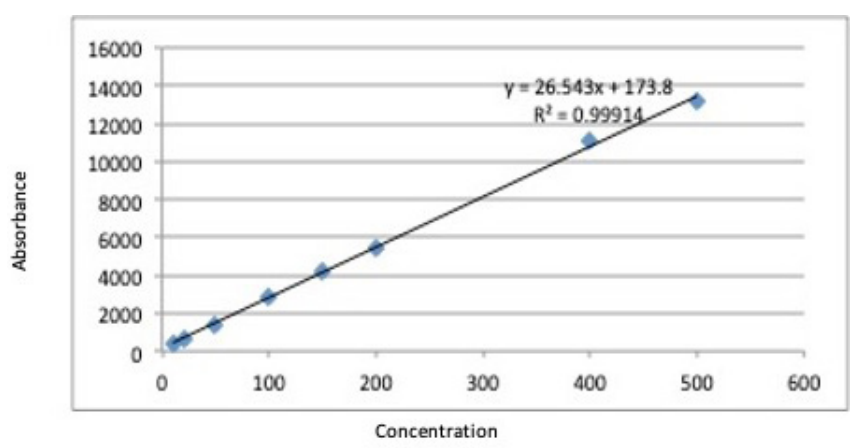

Figure 2. Calibration curve of the rutin

\section{DISCUSSION}

It is a well-known fact that Scorzonera species are beneficial in many medical indications such as, common cold (such as mucolytic, diuretic and antipyretic), wound healing and against acute hepatotoxicity, arteriosclerosis, kidney diseases, hypertension, diabetes mellitus, rheumatism, pain $(7,12,22,23)$. Studies related to Turkish folk medicine have mentioned that many of the Scorzonera species have antidiabetic properties $(7,13)$ and a single recent study has been reported $S$. cinerea radical leaves have an antidiabetic effect by inhibiting $\alpha$-amylase and $\alpha$-glucosidase and increasing the insulin level in diabetic rats (24). Although all that background a study that examines the antidiabetic properties, with an up-to-date method, of many Scorzonera species was not found. In the current study, Scorzonera species were evaluated for their antidiabetic potentials on alloxan induced diabetic mice in order to clarify their 
traditional usage as antidiabetic medicinal plants. $S$. sublanata and S. cana var. jacquiniana were found to have blood glucose lowering effect. Rutin is one of constituents of various Scorzonera species which was reported to have potential antidiabetic activity previously (16). In present study antidiabetic activity of rutin was investigated and compared with previous studies. Furthermore, this study was tried to find a relationship with rutin content in Scorzonera species and antidiabetic activity.

Rutin is a common flavonoid that is broadly consumed from plant-derived beverages and foods as traditional and in folkloric medicine. Rutin is mentioned to exhibit fundamental pharmacological activities, including anti-inflammation, anti-diabetic, anti-adipogenic and anti-oxidation (25). For example, after rutin treatment to streptozotocin-induced diabetic rats for 45 days at $100 \mathrm{mg} / \mathrm{kg}$ resulted in decreasing plasma glucose and increasing insulin levels along with the restoration of glycogen content and increasing activities of carbohydrate metabolism enzymes (26). Histopathological studies have also revealed that rutin has protective effects for the pancreas; expansion of the islets and decreased fatty infiltration of the islets were also observed in treatments with rutin. In the same study, healthy rat parameters were not changed by rutin treatment (17). The underlying mechanism is thought to be decreasing carbohydrate absorption from the small intestine, enhancing insulin secretion by pancreatic b-cell stimulation, increasing tissue glucose uptake and inhibition of tissue gluconeogenesis (16).

Type 2 diabetes is the most prevalent form of diabetes mellitus and it is shown that rutin administration to these patients improve significant parametres such as plasma glucose, insulin level and and antioxidant status of liver (2729). Rutin treatment displayed antidiabetic activity in high fat diet + streptozotosin induced diabetic rats by affecting many cellular events participating in the etiology of type 2 diabetes. Rutin descreased plasma glucose, increased body weight, decreased glycosylated hemoglobin significantly, and its effect is comparable to pioglitazone (a clinically effective new generation antidiabetic drug). Furthermore, rutin reduced intracellular pro-inflammatory cytokines such as IL-6 and TNF-alpha, which are intimately associated with insulin resistance, could result in improved insulin receptor substrate phosphorylation (29).

Diabetic patients with combined dyslipidemia are common; many diabetic patients have elevated low-density lipoprotein (LDL) and triglyceride as well as the reduced level of highdensity lipoprotein (HDL) (28). The study that is mentioned above stated that after 3 weeks of rutin administration a significant decrease in plasma total cholesterol, total triglyceride, $L D L$, very low density lipoprotein (VLDL) and an increase in HDL levels were recorded in high fat diet + streptozotosin induced diabetic rats (29). Likewise, in another study after 3 weeks of oral administration of rutin to streptozotocin-induced diabetic rats, reduced levels of lipids in plasma and tissues, increased HDL-cholesterol, decreased LDL- and VLDL-cholesterol levels of plasma were observed
(30). Moreover, atherosclerosis and cardiovascular diseases are more prevalent in diabetic patients $(28,31)$. Studies are claimed that rutin also protect and improve myocardial dysfunction, oxidative stress, apoptosis and inflammation in the hearts of the diabetic rats (27).

In the current study it is shown that rutin has significant antidiabetic activity, this data is consistent with the literature. However, antidiabetic activity was not directly associated with rutin content of the plant extracts. S. sublanata and S. cana var. jacquiniana displayed remarkable antidiabetic activity while they contain rutin in low amount. According to the HPLC results all investigated Scorzonera species contain mainly phenolic structures as flavonoid derivatives, some chlorogenic acid derivatives and other constituents. From the chemical point of view, the Scorzonera species have been subjected to intensive studies which have led to the isolation of several types of compounds. Previously, a number of compounds such as dihydroisocoumarines, bibenzyl derivatives, flavonoids, lignans, stilbene derivatives, quinic and caffeic acid derivatives, sesquiterpene, sesquiterpene lactones and triterpenes have been isolated from the Scorzonera species and many of the isolated compounds have been identified as new structures (8-10,12,22-24,32,33). In current study none of the investigated Scorzonera species aerial parts except $S$. cana var. jacquiniana (Syn. Podospermum canum) have been analysed for their chemical contents. S. cana var. jacquiniana contains phenolic compounds and flavonoids as following; arbutin, 6'-O-caffeoylarbutin, cichoriin, 3,5-dicaffeoylquinic acid methyl ester, apigenin-7-O-B-glucoside, luteolin-7$O$ - 8 -glucoside, apigenin-7-O-B-rutinoside, isoorientin, orientin, vitexin, procatechuic acid, and 4-hydroxy-benzoic acid 4-(6-O- $\alpha$-rhamnopyranosyl- 6 -glucopyranosyl) benzyl ester in its aerial parts (28). According to the phytochemical investigation results of the current study, also previous researches on Scorzonera species it could be suggested that the antidiabetic activities of these extracts based on synergistic effect of the phenolics, and especially flavonoids with other constituents.

Our findings should be interpreted in light of several limitations. The main limitation of the study that should be mentioned was the lack of healthy control and positive control groups. Since it is the first study that investigate antidiabetic activity for many evaluated Scorzonera species, authors have only planned to compare with diabetic control group. Moreover, antidiabetic activity was only evaluated with single parameter such as prolonged effect of the Scorzonera species on blood glucose was not evaluated, this can be counted as a limitation of the study. Regarding to preliminary results of this study, active extracts and possible other active contents will be evaluated in further detailed studies.

\section{CONCLUSION}

In conclusion, the antidiabetic usage of the Scorzonera species in Turkish folk medicine has been indicated by the current study. S. sublanata displayed the highest antidiabetic 
activity, followed by $S$. cana var. jacquiniana aerial part extracts. Rutin, also exhibited the significant reducing effect on blood glucose levels in alloxan diabetic mice as one of the constituents of some Scorzonera species, in the current study. Rutin content was not found to be correlated with the activity of the tested extracts. On the other hand, HPLC analyses have revealed that the tested Scorzonera species contain many phenolic compounds and flavonoids which were not clarified in detail. Therefore, further well-designed studies are needed to enlight the responsible compounds as well as their mechanisms in the treatment of diabetes mellitus.

\section{Conflict of interest}

The authors declare that they have no competing interests.

\section{Funding}

This research received no external funding.

\section{REFERENCES}

[1] Bahmani M, Zargaran A, Rafieian Kopaei M, Saki K. Ethnobotanical study of medicinal plants used in the management of diabetes mellitus in the Urmia, Northwest Iran. Asian Pac J Trop Med. 2014; 7(S1):348-354.

[2] Choudhury $H$, Pandey M, Hua CK, Mun CS, Jing JK, Kong L, Ern LY, Ashraf NA, Kit SW, Yee TS, Pichika MR, Gorain B, Kesharwani $P$. An update on natural compounds in the remedy of diabetes mellitus: A systematic review. J Tradit Complement Med. 2018; 3:361-376.

[3] Tripathi V, Verma J. Different models used to induced diabetes: A comprehensive review. Int J Pharm Pharm Sci. 2014; 6(6):2932.

[4] American Diabetes Association. Classification and Diagnosis of Diabetes. Diabetes Care. 2015; 35(Suppl.1):8-16.

[5] Arumugam G, Manjula P, Paari N. A review: Anti diabetic medicinal plants used for diabetes mellitus. J Acute Dis. 2013; 2(3):196-200.

[6] Coşkunçelebi K, Makbul S, Gültepe M, Okur S, Güzel ME. A conspectus of Scorzonera s.I. in Turkey. Turk J Botany. 2015; 39:76-87.

[7] Baytop T. Turkiye'de Bitkiler ile Tedavi (Phytotherapy in Turkey, Past and Present). Istanbul: Nobel Publishers; 1999.

[8] Granica S, Lohwasser U, Jöhrer K, Zidorn C. Qualitative and quantitative analyses of secondary metabolites in aerial and subaerial of Scorzonera hispanica L. (black salsify). Food Chem. 2015; 173:321-331.

[9] Wang Y, Wray V, Tsevegsuren N, Lin W, Proksch P. Phenolic compounds from the Mongolian medicinal plant Scorzonera radiata. Zeitschrift fur Naturforsch - Sect C J Biosci. 2012; 67:135-143.

[10] Zidorn C, Ellmerer-Müller E, Stuppner H. Sesquiterpenoids from Scorzonera hispanica L. Pharmazie. 2000; 55(7):550-551.

[11] Dolota A, Dabrowska B, Radzanowska J. Chemical and sensory characteristics of some Scorzonera (Scorzonera hispanica L.) cultiars. Acta Hortic. 2005; 682:527-534.
[12] Ajebli M, Amssayef A, Eddouks M. Assessment of antihyperglycemic effect and acute yoxicity of the aqueous Scorzonera undulata extract in rats. Endocr Metab Immune Disord Drug Targets. 2020; 20. doi: 10.2174/187.153.032066 6.200.729151133.

[13] Cakilcioglu U, Turkoglu I. An ethnobotanical survey of medicinal plants in Sivrice (Elazig-Turkey). J Ethnopharmacol. 2010; 132:165-175.

[14] Polat R, Cakilcioglu U, Satil F. Traditional uses of medicinal plants in Solhan (Bingöl - Turkey). J Ethnopharmacol. 2013; 148(3):951-963.

[15] Dalar A. Plant Taxa Used in the Treatment of Diabetes in Van Province, Turkey. Int J Second Metab. 2018; 5(3):170-184.

[16] Ghorbani A. Mechanisms of antidiabetic effects of flavonoid rutin. Biomed Pharmacother. 2017; 96:305-312.

[17] Stanley Mainzen Prince P, Kamalakkannan N. Rutin improves glucose homeostasis in streptozotocin diabetic tissues by altering glycolytic and gluconeogenic enzymes. J Biochem Mol Toxicol. 2006; 20(2):96-102.

[18] Kumar S, Kumar D, Deshmukh RR, Lokhande PD, More SN, Rangari VD. Antidiabetic potential of Phyllanthus reticulatus in alloxan-induced diabetic mice. Fitoterapia. 2008; 79:21-23.

[19] Ozbek H, Acikara OB, Keskin I, Kirmizi NI, Ozbilgin S, Oz BE, Kurtul E, Ozrenk BC, Tekin M, Saltan G. Evaluation of hepatoprotective and antidiabetic activity of Alchemilla mollis. Biomed Pharmacother. 2017; 86:172-176.

[20] Sun C, Li X, Liu L, Canet MJ, Guan Y, Fan Y, Zhou Y. Effect of fasting time on measuring mouse blood glucose level. Int J Clin Exp Med. 2016; 9(2):4186-4189.

[21] Özbek H, Yılmaz BS. Anti-inflammatory and hypoglycemic activities of alpha-pinene. Acta Pharm Sci. 2017; 55(4):7-14.

[22] Küpeli Akkol E, Acikara OB, Süntar I, Citolu GS, Kele H, Ergene B. Enhancement of wound healing by topical application of Scorzonera species: Determination of the constituents by HPLC with new validated reverse phase method. J Ethnopharmacol. 2011; 137:1018-1027.

[23] Özbek H, Bahadır Acıkara Ö, Keskin i, Kirmizi Ni, Yiğitbaşi T, Şakul AA, Iscan Saltan G. Preclinical Evaluation of Scorzonera spp. Root Extracts and Major Compounds against Acute Hepatotoxicity Induced by Carbon Tetrachloride. Indian J Pharm Sci. 2017; 79(5):715-723.

[24] Temiz MA. Antioxidant and antihyperglycemic activities of Scorzonera cinerea radical leaves in streptozocin-induced diabetic rats. Acta Pharm. 2021; 71. doi: 10.2478/acph-20210045

[25] Chua LS. A review on plant-based rutin extraction methods and its pharmacological activities. J Ethnopharmacol. 2013; 150:805-817.

[26] Kamalakkannan N, Prince PS. Antihyperglycaemic and antioxidant effect of rutin, a polyphenolic flavonoid, in streptozotocin-induced diabetic wistar rats. Basic Clin Pharmacol Toxicol. 2006; 98:97-103.

[27] Vinayagam R, Xu B. Antidiabetic properties of dietary flavonoids: a cellular mechanism review. Nutr Metab (Lond). 2015; 12(60):1-20.

[28] Grundy SM, Benjamin IJ, Burke GL, Chait A, Eckel RH, Howard BV, Mitch W, Smith SC Jr, Sowers JR. Diabetes and Cardiovascular Disease a Statement for Healthcare Professionals from the American Heart Association. Circulation. 1999; 100:11341146 . 
[29] Niture NT, Ansari AA, Naik SR. Anti-hyperglycemic activity of rutin in streptozotocin-induced diabetic rats: An effect mediated through cytokines, antioxidants and lipid biomarkers. Indian J Exp Biol. 2014; 52:720-727.

[30] Prince PSM, Kannan NK. Protective effect of rutin on lipids, lipoproteins, lipid metabolizing enzymes and glycoproteins in streptozotocin-induced diabetic rats. J Pharm Pharmacol. 2006; 58(10):1373-1383.

[31] Leon BM, Maddox TM. Diabetes and cardiovascular disease: Epidemiology, biological mechanisms, treatment recommendations and future research. World J Diabetes 2015. 2015; 6(13):1246-1258.

[32] Akkol EK, Šmejkal K, Kurtul E, Ilhan M, Güragac FT, İşcan GS, Acıkara ÖB, Cvačka J, Buděšínský $M$. Inhibitory activity of Scorzonera latifolia and its components on enzymes connected with healing process. J Ethnopharmacol. 2019; 245:112168.

[33] Bahadır Acıkara Ö, Ilhan M, Kurtul E, Šmejkal K, Küpeli Akkol E. Inhibitory activity of Podospermum canum and its active components on collagenase, elastase and hyaluronidase enzymes. Bioorg Chem. 2019; 93:103330.

How to cite this article: Sakul AA, Kurtul E, Ozbek H, Kirmizi NI, Bahtiyar BC, Saltan Iscan G, Acikara OB. Evaluation of Antidiabetic Activities of Scorzonera Species on Alloxan-Induced Diabetic Mice. Clin Exp Health Sci 2021; 11: 74-80. DOI: 10.33808/ clinexphealthsci.797747 